\title{
Factors Influencing Consumer Preference of Fresh Potato Varieties in Maine
}

\author{
John M. Jemison Jr • Peter Sexton • Mary Ellen Camire
}

Published online: 5 September 2008

(C) Potato Association of America 2008

\begin{abstract}
Tuber color and appearance are important factors that influence consumer decision-making. In order to evaluate which factors are important to consumers in Maine, we surveyed 275 people over two years and four locations to assess fresh potato consumption patterns and to determine how specific potato characteristics influence variety selection. A majority of the study participants were women between the ages of 41 and 60 . Over $60 \%$ of survey participants cooked and consumed fresh potatoes between one and three times per week, and over $84 \%$ of participants reported that baked, mashed, or roasted potatoes were their preferred methods of preparing fresh potatoes. Over $96 \%$ of survey participants expressed that potatoes were a healthy food, although more than a third stated that they limited their carbohydrate consumption. The most important potato characteristics found to influence fresh potato purchase were skin quality and place of origin with $30 \%$ and $41 \%$ of participants giving each of these factors the highest possible score. Participants appear to recognize the importance of the industry, and many expressed interest in buying locally grown potatoes. Skin quality was the most important characteristic influencing preference for whole white and yellow skin varieties (seven of eight varieties) evaluated in the study. Skin quality also influenced variety selection of
\end{abstract}

The online version of the original article can be found at http://dx.doi.org/ 10.1007/s12230-008-9017-3.

J. M. Jemison Jr $(\bowtie)$

495 College Avenue, Orono, ME 04473, USA

e-mail: jemison@maine.edu

P. Sexton

P.O. Box 727, Houlton Road, Presque Isle, ME 04769, USA

M. E. Camire

University of Maine, 105 Hitchner Hall, Orono, ME 04469, USA whole red skin potatoes in five of eight varieties. This suggests that more research is needed to develop varieties and production methods that produce attractive blemishfree potatoes. When participants could see the potato flesh, it significantly influenced variety selection with all potatoes. Flesh color was the most important characteristic of halved potatoes with 13 of 14 possible varieties. Pictures showing potato flesh color may also help retail sales of table stock varieties. The yellow fleshed varieties with white and purple skins were quite popular, but yellow flesh, red skin varieties were less popular.

Resumen El color y apariencia del tubérculo son factores importantes que tienen influencia en la decisión del consumidor. Con el fin de evaluar cuales son los factores importantes para el consumidor en Maine, hemos encuestado 275 personas en dos años, en cuatro localidades para evaluar los patrones de consumo de papa fresca y para determinar como influencia las características específicas de la papa sobre la selección de variedades. Una mayoría de los participantes fueron mujeres entre 41 y 60 años de edad. El $60 \%$ de los participantes encuestados usan y consumen papa fresca una a tres veces por semana y el $84 \%$ de los participantes reportaron que las prefieren horneadas, en puré o asadas. Más del $96 \%$ de los encuestados expresaron que la papa es un alimento sano, aunque más del tercio expresaron que ellos limitan su consumo de carbohidratos. Las características más importantes de la papa que influencia la compra fueron la calidad de la cáscara y el lugar de origen con el 30 y $41 \%$ de participantes dándole a estos factores el más alto puntaje posible. Los participantes parecen reconocer la importancia de la industria y muchos expresaron su interés en comprar papa cultivada localmente. La calidad de la cáscara fue la característica más importante que influenció en las preferencias, para varie- 
dades de cáscara completamente blanca o amarilla (siete de ocho variedades) evaluadas en el estudio. La calidad de la cáscara también influenció en la selección de variedades de cáscara completamente roja en cinco de ocho variedades. Esto sugiere que se necesita mayor investigación para desarrollar variedades y métodos de producción que lleven a la obtención de papas atractivas sin manchas. Cuando los participantes pudieron ver la pulpa del tubérculo, esto influenció significativamente la selección de variedades. El color de la pulpa fue la característica más importante de papa cortada con 13 de 14 variedades posibles. Fotos que muestran el color de la pulpa pueden ayudar a la venta al por menor de las variedades de consumo en fresco. Las variedades de pulpa amarrilla con cáscara blanca y púrpura fueron muy populares, y las de pulpa amarilla con cáscara roja fueron las menos populares.

The Resumen was inadvertently omitted from this article which was published in vol. 85 no. 2, p. 140. It is reproduced here along with the English abstract.

Springer regrets the error. 